\title{
Efficacy of umbilical cord tissue derived mesenchymal stem cells in the management of advanced rheumatoid polyarthritis
}

\section{Introduction}

Rheumatoid arthritis (RA) is a systemic autoimmune disease characterized with chronic inflammatory synovitis and progressive joint destruction that causes severe morbidity and mortality. However, once a joint is damaged or deformed, it cannot be repaired. Auto-reactive $\mathrm{T}$ cells and inflammatory cytokines such as TNF alpha play a pivotal role in the pathogenesis of synovial inflammation and irreversible joint destruction, induced by the production of matrix metalloproteinases as well as the maturation and activation of osteoclasts. Therefore, a novel treatment strategy aimed to control inflammation and promote osteochondral regeneration is a prerequisite. ${ }^{1-3}$ Pre-clinical researches have demonstrated that the MSCs derived from bone marrow, adipose tissue, synovium and umbilical cord, all suppress the functions of different immune cells and simultaneously express regenerative effect thus raising the possibility of new therapeutic approach for autoimmune diseases including RA. ${ }^{4}$

As of yet, the most common source of MSCs has been bone marrow. However, aspirating bone marrow is an invasive procedure. In addition, the number and the differentiating potential of bone marrow MSCs (BM-MSCs) decrease with age. ${ }^{5,6}$ In contrast, the umbilical cord is a postnatal organ discarded after birth. The collection of umbilical cord MSCs (UC-MSCs) does not require any invasive procedure. In addition to the well-documented self-renewal and multipotent differentiation properties, UC-MSCs possess immunoregulatory capacities that have been permissive to allogeneic transplantation. ${ }^{7}$ In this study, we reported our findings of the therapeutic effect of Umbilical cord tissue derived MSCs in Rheumatoid Polyarthritis.

\section{Case report}

A 31 year old lady presented with severe pain and swelling both knee, both wrist, right elbow and all small joints of hand. She consulted her general practitioner multiple times with increasing levels of pain and disability before she was eventually diagnosed with rheumatoid polyarthritis.

Biochemical investigations showed increased erythrocyte sedimentation rate (ESR) and C-reactive protein (CRP) and RA test positive. MRI of the knee showed decreased thickness of articular cartilage,synovial thickening increased and joint effusion present. She was on Disease-modifying antirheumatic drugs (DMARDS) like sulphasalazine, chloroquine, methotrexate and corticosteroids for the last 7 years. In spite of taking above treatment she developed complications in the form of secondary arthritis with osteopenia in multiple joints. So we have decided to treat the patient with mesenchymal stem cell therapy. Mesenchymal stem cells were isolated from umbilical cord tissue preserved of her son. 150 million Human umbilical cord tissue derived MSC were transfused through intravenous route while 10 million MSC were given intra articular in
Volume 4 Issue I - 2018

\author{
BS Rajput,' Rani James, ${ }^{2}$ Sushma Kudari, ${ }^{2}$ \\ Kaushik D Deb² \\ ${ }^{1}$ Criticare hospital and research centre, Mumbai \\ ${ }^{2}$ Dipon Ed Biolntelligence, India
}

\begin{abstract}
Correspondence: Kaushik Deb, DiponEd Biolntelligence LLP \#60/A, 2nd Floor, Karnataka Bank Building Jigani Road, Bomasandra Industrial Area Bangalore, Karnataka 560099, India, Tel +91990052 0141, +9(0)80 27835022. Email deb@diponed.com
\end{abstract}

Received: February 08, 2018 | Published: February 27, 2018

both knee, 5 million in both wrist and right elbow. Total 6 sessions were done over a period of 6 months. The supply of cells was done by Cryo VIVA International. The study was approved by Independent Ethics Committee. Duly informed consent was taken from the patient in her understandable language.

The primary objective of the study was to evaluate the effectiveness mesenchymal stem cell therapy in improving the signs \& symptoms of subjects with Rheumatoid Polyarthritis by assessing decrease in pain, stiffness and physical functions from baseline to the completion of therapy using WOMAC scale, ADL Score along with 6 Minute Walking Distance (MWD). WOMAC score is a widely used, proprietary set of standardized questionnaires used by health professionals to evaluate the condition of patients with osteoarthritis of the knee and hip, including pain, stiffness, and physical functioning of the joints. The WOMAC covers 3 dimensions: pain, stiffness, and physical function. The data were standardized to a range of values from 0 to 100 , with 0 representing the best health status and 100 the worst possible status. ${ }^{8}$ Activities of Daily Living (ADLs) are tasks related to personal care. The ADL score tests out different tasks like: transfer, bed mobility, toileting eating capabilities of the patient and scored at a ranges from 0 (independent) to 4 (total dependence)

\section{Results}

\section{a. Clinical outcome}

After 6 months of treatment with mesenchymal stem cells, pain and swelling of both knee reduced markedly. Mobility of the limbs increased. Stiffness of right elbow disappeared completely. Stiffness of both wrist decreased by 50 percent and mobility is increased. The pain score decreased overall. 
Figure 1 showed the variation in the WOMAC scores, which decreased significantly week 4 onwards, for all the three parameters Pain, Stiffness and Physical disability. The difference got more significant as the trial progressed from week 4, week 8, week 12 and the follow up period of week-24.

Figure 2 showed significant change in the distance walked in 6 minutes. This was consistent as after continuous exercise (continuous

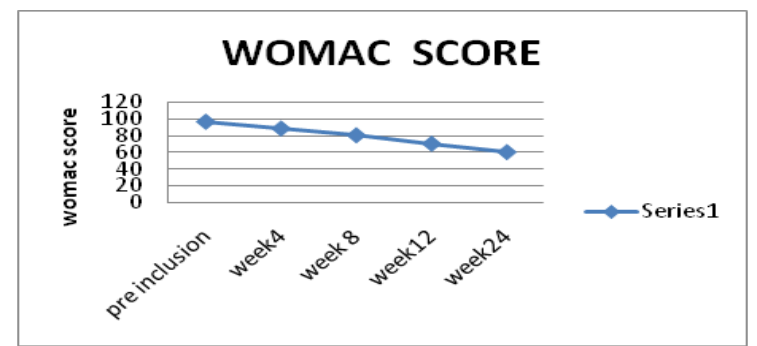

Figure I Change from baseline in WOMAC Scores. $X$ axis represents duration in weeks. Y axis represents Total WOMAC scores.

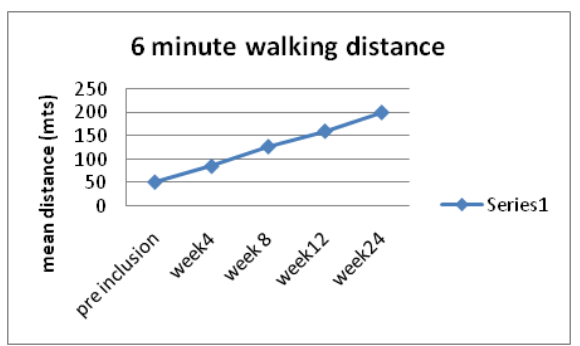

Figure 2 Change from baseline in Distance Walked in 6 Minutes. $X$ axis represents duration in weeks. $Y$ axis represents the mean distance coved in meters.

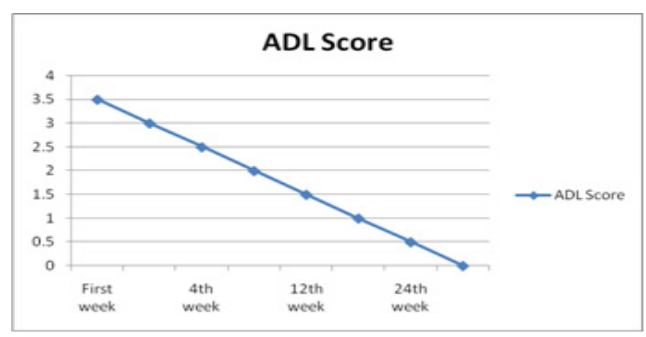

Figure 3 Change from baseline in $A D L$ scores. $X$ axis represents duration in weeks. $Y$ axis represents Total ADL scores.

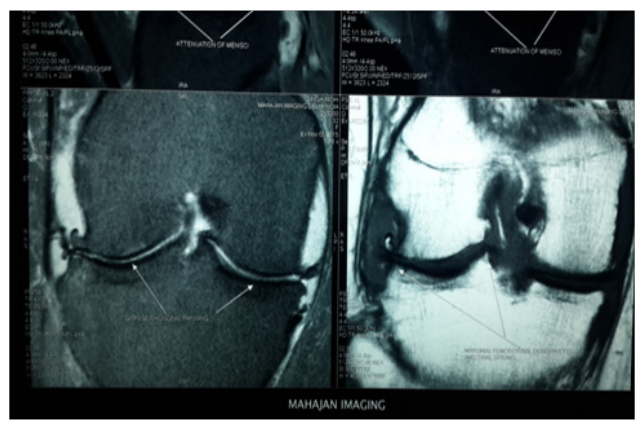

Figure 4A pre-stemcell transplantation walking for 6 minutes), increase in the vital parameters was natural and physiological. Figure 3 showed changes in ADL scores.

Biochemical test showed decreased level of ESR, CRP and Rheumatoid Titer. X-rays showed less Osteopenia. MRI of knee showed increased thickness in articular cartilage with reduction in synovial thickening and joint effusion. There was visible repair of articular cartilage breach Figures (4-6B).

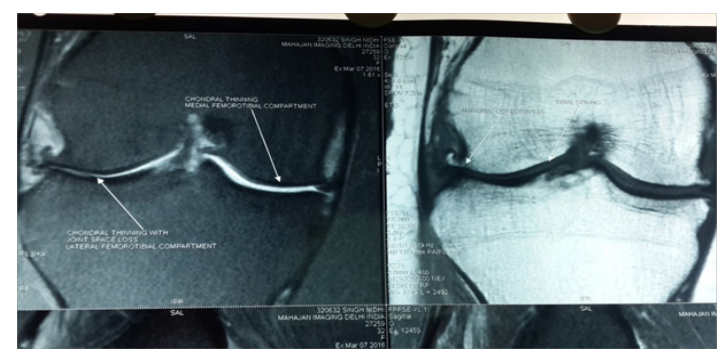

Figure 4 (B) Post-stemcell transplantation.

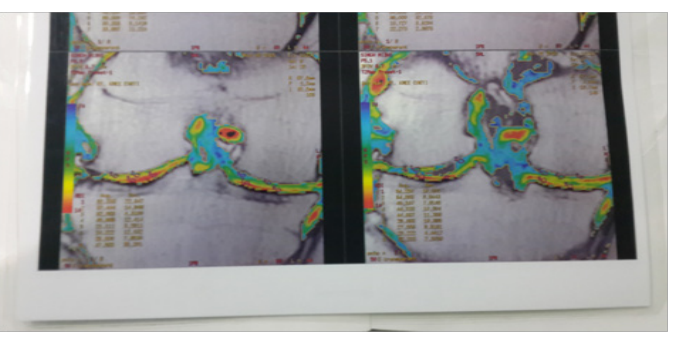

Figure 5A pre-stemcell transplantation

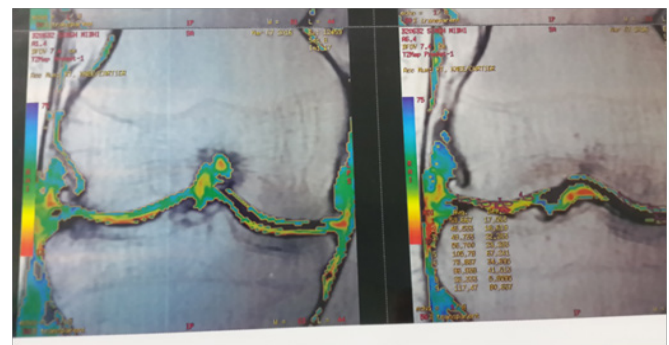

Figure 5 (B) Post-stemcell transplantation.

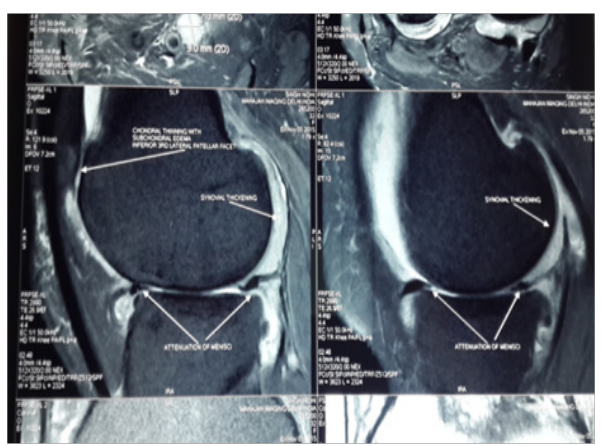

Figure 6 (A) Pre-stemcell transplantation. 


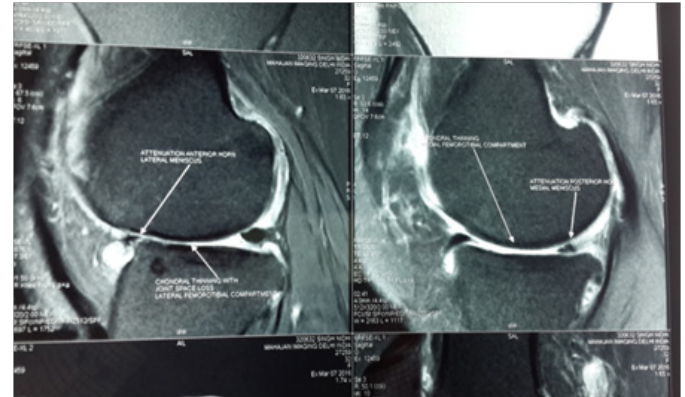

Figure 6 (B) post stemcell transplantation

\section{Discussion}

Despite the growing interest in stem cell transplantation only few trials, investigating the efficacy of umbilical cord tissue derived mesenchymal stem cells (UC)-MSCs in the treatment of RA have been published. MSCs clearly are immunosuppressive and immunomodulatory. The remarkable potential of MSCs to modulate the host immune response, mainly by inhibiting the proliferation of $\mathrm{T}$ lymphocytes, introduced the possibility that they might be effective in inflammatory arthritis where the T-cell response is prominent Clinical data available on the web indicate that MSCs administration significantly reduced pain and degenerative lesions associated with Rheumatoid Arthritis. ${ }^{9-11}$

Regeneration of the damaged joints of patients with RA could potentially be induced by MSCs, based on the following: 1) MSCs can differentiate into osteoblasts in the presence of inflammatory cytokines, which are abundantly present in the inflamed synovium, 2) MSCs produce high amounts of osteoprotegerin, which efficiently suppresses RANKL/RANK pathway-mediated osteoclastogenesis, and 3) MSCs have immunosuppressive properties, making them further suitable for the treatment of RA. ${ }^{11}$

The WOMAC scores in our study demonstrated significant improvement in pain, stiffness and physical disability. Similarly improvement was seen on ADL scores. The distance covered in 6 minutes too increased noticeably and was consistently more than that seen at the baseline. Furthermore, no adverse effects were noted in the trial. The scores reduced significantly in all the parameters under consideration, hence substantiating the efficacy of mesenchymal stem cells.

Taken together, the results of this study suggest that umbilical cord tissue (UC) derived mesenchymal stem cells (MSCs) could be effective in the treatment of several autoimmune diseases. The conclusion of this study was that UC -MSCs transplantation is as effective as chondrocytes for cartilage repair and requires less knee surgery, reduces costs and minimizes donor-site morbidity. The therapeutic application of mesenchymal stem cells represents a promising approach in rheumatic diseases. Thus, MSC-based therapy may serve as an alternative method for the treatment of mild to moderately damaged joints in Rheumatoid Arthritis.

\section{Acknowledgements}

\section{None}

\section{Conflict of interest}

The author declare there is no conflict of interest.

\section{References}

1. Burmester Gr, Kivitz Aj, Kupper H, et al. Efficacy and safety of ascending methotrexate dose in combination with adalimumab: the randomised concerto trial. Ann Rheum Dis. 2015;74(6):1037-1044.

2. Furst DE, Emery P. Rheumatoid arthritis pathophysiology: update on emerging cytokine and cytokine-associated cell targets. Rheumatology (Oxford). 2014;53(9):1560-1569.

3. McInnes IB, SCHETT G. The pathogenesis of rheumatoid arthritis. $N$ Engl J Med. 2011;365(23):2205-2219.

4. El-Jawhari JJ, YM El-Sherbiny, EA Jones, et al. Mesenchymal stem cells, autoimmunity and rheumatoid arthritis. QJM. 2014;107(7):505-514.

5. Chiang EY, Kolumam GA, Yu X, et al. Targeted depletion of lymphotoxinalpha-expressing TH1 and TH17 cells inhibits autoimmune disease. Nat Med. 2009;15(7):766-773.

6. Rao MS, Mattson MP. Stem cells and aging: expanding the possibilities. Mech Ageing Dev. 2001;122(7):713-734.

7. Baksh D, Yao R, Tuan RS. Comparison of proliferative and multilineage differentiation potential of human mesenchymal stem cells derived from umbilical cord and bone marrow. Stem Cells. 2007;25(6):1384-1392.

8. Nauta AJ, Fibbe WE. Immunomodulatory properties of mesenchymal stromal cells. Blood. 2007;110(10):3499-3506.

9. Quintana J, Escobar A, Arostegui I, et al. Health-Related Quality of Life and Appropriateness of Knee or Hip Joint Replacement. Arch Intern Med. 2006;166(2):220-226

10. Trivanovic D, Kocic J, Mojsilovic S, et al. Mesenchymal Stem Cells Isolated from Peripheral Blood and Umbilical Cord Wharton's Jelly. Srp Arh Celok Lek. 2013;141(3-4):178-186.

11. Le Blanc K, Rasmusson I, Sundberg B, et al. Treatment of severe acute graft-versus-host disease with third party haploidentical mesenchymal stem cells. Lancet. 2004;363(9419):1439-1441. 